\title{
Self-Folding Non-Invasive Miniature Robots: Progress and Trend in the Biomedical Field
}

\author{
Vienna Parnell \\ The Harker School, 500 Saratoga Ave, San Jose, CA 95129. \\ Corresponding author. E-mail: 22viennap@gmail.com
}

Received: Mar. 29, 202I; Accepted: Sep. 9, 2021; Published: Oct. 25, 2021

Citation: Vienna Parnell, Self-Folding Non-Invasive Miniature Robots: Progress and Trend in the Biomedical Field. Nano Biomed. Eng., 202I, I3(4): 329-343.

DOI: $10.5101 /$ nbe.v13i4.p329-343.

\begin{abstract}
Developments in surgery have been geared toward minimizing the invasiveness of the procedure to improve both the treatment itself and the patient's postoperative wellbeing. As such, attention has been directed toward reducing human error and miniaturizing clinical devices by developing smaller devices and robotic systems. While there have already been significant advancements in this area, apparatus can further benefit from being foldable, expandable, and further condensable. By promoting these characteristics, origami engineering, which extrapolates the fundamental principles of paper folding to real-world projects, has become increasingly prevalent in the biomedical field. This paper reviews the field of origami engineering, its fundamental mechanical and mathematical properties, and the recent progress in specific research areas. Then, this paper discusses several devices that have emerged over the past decade in detail based on their characteristics and implementations. Finally, this paper addresses the technical challenges and general research trend of self-folding non-invasive miniature robots.
\end{abstract}

Keywords: Origami engineering, Microrobots, Noninvasive, Self-folding, Biocompatible

\section{Introduction}

Advancements in surgery have resulted in increasingly autonomous and miniaturized apparatus. As a consequence, condensed robotic components have become progressively relevant in the biomedical field, diffusing across a wide range of surgical operations [1]. Despite potentially allowing for more accurate treatment, this shift has introduced complications regarding cost and usefulness for certain ailments over established practices, stemming from the size of the device and flexibility of conventional robotic systems [2], [3].

Both of these concerns can be addressed through the implementation of origami principles in robotic systems. Drawing inspiration from the traditional art of paper folding, applications of origami in engineering can be characterized by their foldability and versatility [4]. In the context of biomedical applications and robotic systems, origami principles can support the development of a small, self-actuating mechanism that can adapt to its surroundings and conduct noninvasive tasks [5].

By applying the properties of rigid-foldability and pliability to improve MIS, origami has the potential to revolutionize the biomedical industry by proposing even safer and more effective alternatives to current procedures. Of particular interest are self-folding medical microrobots, which are designed to traverse bodily conditions while performing tasks internally. In 
this paper, we review the clinical benefits of origamiinspired microrobots, as well as their current progress and trend in improving current biomedical procedures.

\section{Origami Engineering}

The art of "paper folding," commonly known as 'origami,' has been practiced for over five centuries as a form of artistic expression. From only a flat sheet, the designer can form intricate structural wonders through simple folds and occasionally incisions. Originating in Japan and China, origami did not rise in prevalence in the Western world until the 20th century through publications providing instructions for the reader to follow for recreational intentions [6]. In addition, the term "kirigami," meaning "paper-cutting” was also coined; as the name suggests, kirigami involves creating incisions in the fabrication material in addition to traditional folding [7]. This art allows the designer more freedom, as he or she can manipulate the material beyond simple folds.

Other than being an enjoyable pastime, origami has also emerged in academic studies. Dating back to the early $19^{\text {th }}$ century, the mathematics of origami was used in supporting geometric proofs [8]. Now, origami has fully transcended its traditional purposes and is now valued as an integral part of real-world engineering projects due to its various unique characteristics. Some principal areas of origami engineering applications include delivery and storage, space, optics, biomedical devices, manufacturing, robotics, and architecture [5].

\section{Mechanical and mathematical properties}

On a physical level, origami describes the process of transforming a two-dimensional surface into a threedimensional structure [9]. Folding produces several useful mechanical and geometric properties that can be extrapolated from conventional origami art design to engineering projects (Table 1). It is also important to note that these principles are scalable, meaning that they can be achieved and reproduced in a wide range of sizes [10].

To create a three-dimensional origami model, it is first necessary to design a crease pattern or base that will define the ultimate shape of the structure. Tessellations of crease patterns can form complex, three-dimensional structures capable of translational and rotary motion, serving as the basis for a variety of devices in industries involving transportation, manufacturing, and packaging [5]. The Miura-ori pattern, the waterbomb base, the Yoshimura pattern, and the diagonal pattern are common designs that model the rigid-foldability of origami (Fig. 1). When tessellated, these crease patterns can exhibit properties of flat-foldability and rigid-foldability appropriate for different applications, described as follows [5]:

(1) The Miura-ori pattern consists of parallelograms (a)
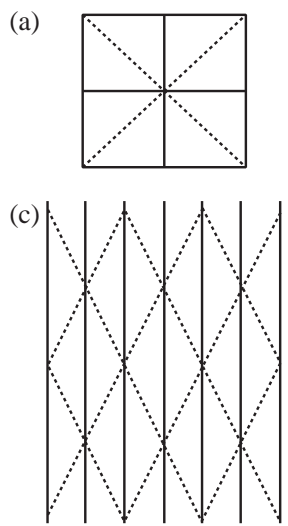

(b)

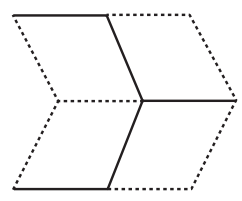

(d)

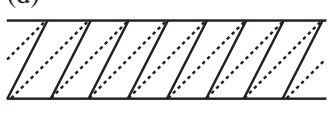

Fig. 1 Four origami crease patterns frequently used in tessellations: (a) Waterbomb base, (b) Miura-Ori pattern, (c) Yoshimura pattern, and (d) diagonal pattern. The solid lines represent mountain folds, while their dashed counterparts represent valley folds [5].

Table 1 Fundamental properties of conventional origami art

\begin{tabular}{|c|c|c|}
\hline Characteristics & Description & Significance \\
\hline Developability & $\begin{array}{l}\text { A three-dimensional origami structure can be developed by folding a two-dimensional } \\
\text { sheet [10]. }\end{array}$ & $\begin{array}{l}\text { Origami structures are restricted to } \\
\text { suitable materials, depending on the } \\
\text { application. }\end{array}$ \\
\hline Flat-foldability & $\begin{array}{l}\text { Origami structure can be folded into a flat configuration with its ultimate thickness } \\
\text { depending on the material. For a vertex to be considered flat-foldable, it has to fulfill the } \\
\text { following conditions [5]: } \\
\text { Kawasaki's Theorem dictates that the total sum of the even alternating angles is equal to } \\
\text { the total sum of the odd alternating angles [5]. } \\
\text { Maekawa's Theorem states that the difference between the number of mountains and } \\
\text { valleys is two [5]. }\end{array}$ & $\begin{array}{l}\text { The structure can occupy a compact } \\
\text { volume while maintaining a constant } \\
\text { surface area. }\end{array}$ \\
\hline Rigid-foldability & $\begin{array}{l}\text { Origami structure can fold continuously along predefined hinges without introducing } \\
\text { facet deformations. Including additional joints increases the degrees of freedom [5], [10]. }\end{array}$ & $\begin{array}{l}\text { The structure can adopt a rigid, } \\
\text { corrugated form while maintaining its } \\
\text { flexibility and strength. }\end{array}$ \\
\hline
\end{tabular}


and is auxetic, meaning that it possesses a negative Poisson's ratio. An intriguing mathematical property of origami-inspired sheets, the Poisson ratio is defined as the ratio of the lateral strain to the longitudinal strain, and a negative value indicates that when the Miuraori pattern is stretched, the material expands in the perpendicular direction. Most notably, this pattern was originally designed for usage in space solar panels, which benefit from being compact at launch and deployable upon arrival in orbit. Other applications implementing this pattern include deformable energy storage devices [11] and deployable arrays [12].

(2) Despite consisting of simple geometry, the waterbomb base actuates along three phases of motion and is flat-foldable. When tessellated, the pattern forms units possessing negative Poisson's ratios between the radial and axial directions. Applications utilizing the waterbomb base pattern include printable robots [13] and artificial stents [14].

(3) The Yoshimura pattern consists of diamonds, and the curve of the material upon folding varies based on the dimensions of the shapes. Tessellations of this pattern are commonly used in folding cylinders, and applications include cylindrical deployable structures [15] and origami antennas [16].

(4) Sharing similar applications with the Yoshimura pattern is the diagonal pattern, which consists of adjacent parallelograms, each divided diagonally along opposite two corners. When tessellated, this pattern is commonly induced under the torsional buckling of a cylindrical structure, allowing it to deform a particular section and shorten in height [17].

Tessellated origami sheets can be bent along the creases, simulating local deformation and altering the global Gaussian curvature without stretching or shrinking the structure's actual material [4]. The Gaussian (intrinsic) curvature represents the product of the maximum and the minimum curvatures at a point, or the principal curvatures (Fig. 2):

$K=\kappa_{1} \cdot \kappa_{2}$

This calculation is made independent of the structure's surroundings, and the sign of the constant Gaussian curvature indicates the type of surface and its geometry. In contrast, the mean (extrinsic) curvature is based on the behavior of the structure in threedimensional space [5], [18].

\section{Applying origami to engineering applications}

Conceptually, applying the fundamental origami
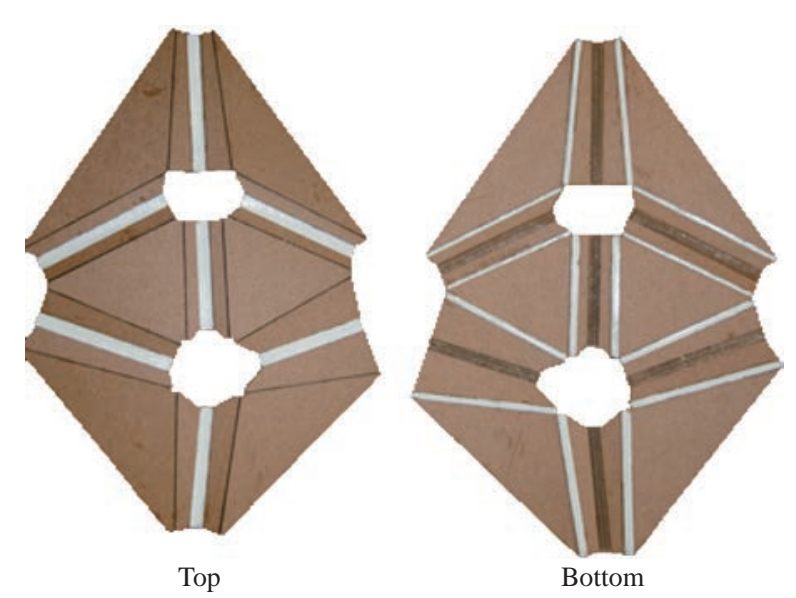

Fig. 2 Positive and negative Gaussian curvature combination structure. Structures Developed by Garza et al. of the Texas A\&M University [84].

principles and designs yields promising results in real-world applications. Being able to condense and expand a structure can facilitate processes ranging from fabrication and storage to deployment and travel. In conventional paper folding, the geometric principles of origami assume a material thickness of zero [8], and similarly, paper is considered to be two-dimensional in mathematical terms [5]. However, real-world devices require much heftier engineering materials depending on their application, as using paper to produce prototypes might introduce significant complications in practical uses [19].

Regardless, these ensuing complications can be surmounted with the usage of appropriate engineering materials and fabrication methods. Some methods that have been explored include connecting the faces of a design using hinges, though a potential issue that arises is over-constrainment. Since several fold lines conjoin at a single vertex, the edges are at risk of intersecting, affecting the practicality of the structure, though decreasing the volume of the valley sides of the edges prevents them from intersecting. Moreover, time and cost may present themselves as additional obstacles to producing origami-inspired designs, and the structure should be durable enough to withstand frequent folding along its hinges [5].

By properly accounting for the addition of thickness, the mechanical properties of origami can be successfully extrapolated to engineering projects, improving existing technology or providing insight into new, imaginative approaches [8]. Practical applications implementing origami engineering include shopping bags [20], shipping containers [21], dynamic sun-shades [22], emergency shelters [23], 
space exploration [24], and interactive humanoids [25] Notably, origami engineering has also been making significant breakthroughs in redeveloping current biomedical procedures, and its numerous fundamental properties contribute to its versatility in the field [26].

\section{Origami-Inspired Biomedical Devices}

Origami-inspired biomedical devices are currently on the rise, given their unique and convenient ability to adopt a compact form to function in constrained spaces, perform non-invasive procedures, and transport substances [5]. As less material is required in fabricating the device, origami-based designs can provide more simplified and affordable treatments for patients. These characteristics allow biomedical devices to operate in vivo more accurately and in previously inaccessible regions. While some biomedical devices perform internally, or in vivo, targeting the cardiovascular system or the immune system, others have external, or ex vivo, purposes, in the form of specialized surgical apparatus [26]. Current applications being explored range according to their targeted area and function (Fig. 3).

\section{Application areas Cardiovascular system}

Applications in the cardiovascular field primarily extrapolate the origami properties of expandability and structural integrity. Devices with these characteristics can navigate and situate in the heart's vessels, where they can support the passageway or perform designated tasks.

One such device that was fabricated using origami folding patterns was the stent graft, a tubular structure that is inserted into a weakened artery to provide support. While stent-grafts are traditionally made with a mesh structure, this design allows for tissue ingrowth to occur, potentially obstructing the passageway in a condition known as restenosis [27]. Kuribayashi et al. of the University of Oxford presented an origamiinspired version of a heart stent [14]. Composed of a single Ni-rich TiNi shape memory alloy foil with hill and valley folds and few gaps and packed in a sheath for transportation, this stent-graft can be manipulated through either thermal or mechanical means, allowing the structure to expand both radially and longitudinally upon arrival at the appropriate location. After creating

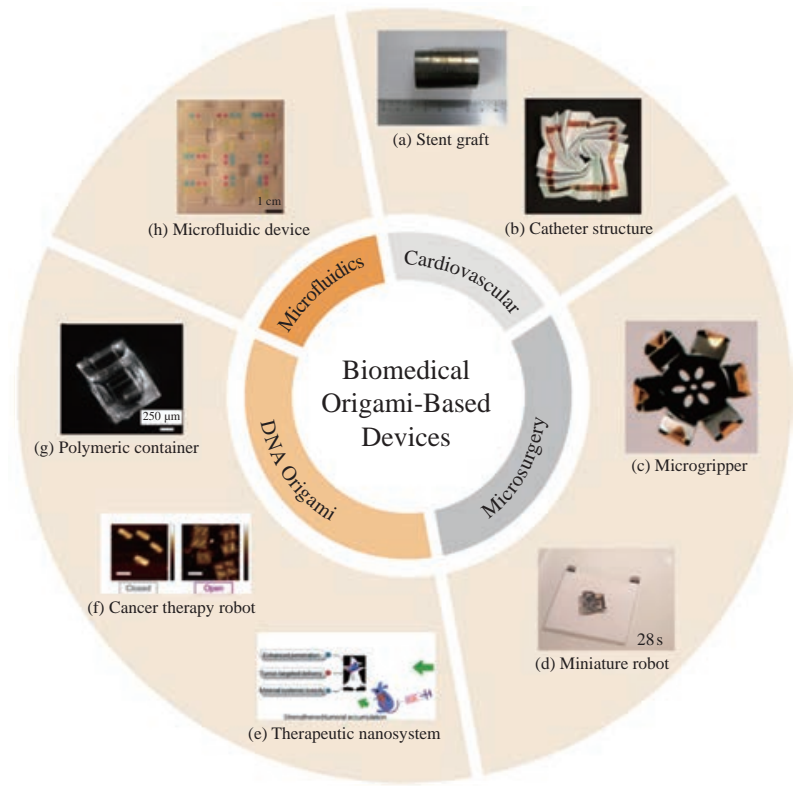

Fig. 3 Biomedical origami-based devices in microfluidic, cardiovascular, microsurgical, and nanoscopic DNA origami applications. (a) Self-deployable stent graft used to support a weakening section of an artery [14]. (b) Expandable catheter structure for capturing images of heart tissue [28]. (c) Microgripper that is triggered to perform in cancerous environments [58]. (d) An untethered, self-folding miniature robot that can perform tasks and disintegrate into the environment [59]. (e) DNA-based origami nanosystem for precise anticancer treatment [38]. (f) Nanorobot that delivers thrombin in the presence of a cancerous tumor [39]. (g) Selffolding polymeric container that encapsulates and delivers therapeutic substances [32].

grooves in the material according to the folding pattern by implementing a negative photochemical etching process, the foils were exposed to a heat aging treatment for two reasons: first, to set the transformation temperatures close to body temperature, and second, to store the original cylindrical shape of the structure in memory. With an acrylic tube representing the catheter and a large acrylic tube imitating an aorta or esophageal lumen, the property of the origami stent graft to self-deploy was experimented in two ways, the first being to test the shape memory effect of the structure. After being cooled using liquid nitrogen to achieve its martensitic state, the graft was measured for its expansion when exposed to warm air. The study found that adjustments to the origami crease pattern and the dimensions of the folds would be necessary to ensure complete recovery of the original shape when the stent is exposed to body temperature. The second experiment evaluated the deployment of the origami stent graft with superelasticity, in which the graft is heated, and due to a release from stress upon removal of the acrylic tube, the structure 
deployment immediately. A compression test found that the graft exhibited the mechanical property of being resistant against a compressive force, making it ideal for its application of supporting a collapsing passageway. While this study demonstrates that the stent-graft can theoretically self-deploy when exposed to heat or external pressure, additional simulations would be needed to evaluate the graft's ability to travel to the appropriate site and expand in bodily conditions. Furthermore, it must be confirmed that the origamiinspired design is more efficient and safer than current options, such that it prevents tissue in-growth and avoids the problem of restenosis in the long run.

Another application related to the cardiovascular system concerned interventional catheter ablation, which is used as an approach to normalizing the heart rhythm of patients with arrhythmia. This treatment utilizes magnetic resonance imaging (MRI) to scan the heart from a location external to the tissue. Taylor, et al. of the University of Georgia proposed an origami expandable mechanism that allows for higher quality image capturing of heart vasculature for early stages of atrial fibrillation treatment [28]. Since the distance between the imaging coil and the tissue is directly related to the quality of the produced image, this study presented a novel expandable origami catheter structure that can be deployed and inserted inside the heart. When fabricated with the proper biocompatible materials, the catheter-based device would allow for the mounting of imaging and treatment electronics. To demonstrate proof of concept, the structure was fabricated using a sheet of polycaprolactone, and a receiver coil was formed by applying copper. An MRI compatibility test was conducted to determine whether the proposed structure would negatively interfere with the MR image, resulting in a lower signal-to-noise ratio (SNR). The results indicated that the maximum SNR reduction was within the acceptable value of $10 \%$ suggested by Chinzei et al., though further studies are necessary to determine how to integrate the catheter with the expandable structure in the fabrication stage [29]. Further, the device's ability to safely conduct real-time cardiac imaging in a simulated in vivo environment should be evaluated.

\section{DNA origami}

Origami properties can be implemented on a nanoscale, allowing for direct interaction with human cells. DNA Origami refers to a method of synthesizing nanoparticles based on Watson-Crick base pairings using arbitrarily folded DNA for potential use in drug delivery and cancer therapy [30]. Through the presence of DNA sequences acting as "staples" and "fasteners," as well as chemical modification of nucleic acids with functional groups, a strand of DNA can maintain the desired structure in three-dimensional space [31].

Fernandes and Gracias of Johns Hopkins University discussed the usage of self-folding polymeric containers in drug delivery applications [32]. Traditional approaches utilize nanoparticles, liposomes, polymer capsules, and micromachined constructs, and origamiinspired packages further ease travel through the body's complex passageways to deliver encapsulated therapeutic substances. These polymeric containers are fabricated using multiple layers of deposited films of a predetermined thickness onto molds or substrates, and in response to a specific stimulus, the films are released. Self-folding can be achieved in various ways, and some of these methods depend on the presence of wires or tethers, while others involve magnetic forces or chemical stimuli. Despite being relatively unexplored, the field of self-folding has many promising applications at scales ranging from centimeters to nanometers, though due to limitations in the 2D nanopatterning of gels and polymers, selffolding at $10-100 \mathrm{~nm}$ size scales is still theoretical. Lithographic patterning, which accurately controls the specifications of the walls of the polymeric containers, may lead to breakthroughs in designing microcarriers [33] and devices capable of the directional [34] and spatio-temporal [35] release of therapeutics.

Another application that implements origami properties concerns therapeutic drug delivery for cancer treatments. Traditional therapeutics involving platinum complexes including cisplatin, oxaliplatin, and carboplatin have strong anticancer abilities at the expense of causing systemic toxicities due to their poor targeting and penetration of tumor cells. Recently, Ruthenium complexes have been considered as a viable alternative, as they result in less severe side effects [36]. Despite these benefits, rutheniumbased treatment still possesses drawbacks regarding its cytotoxic effect, antitumoral mechanism, and stability in aqueous solutions. The field of nanotechnology and nanomedicine, however, has led to developments in anticancer nanosystems, which selectively target cancer cells in a manner less toxic than their conventional chemotherapeutic counterpart through nanocarriers. When incorporated into these systems, DNA origami is advantageous in attacking tumor cells 
due to its well-defined structure, biocompatibility, and efficacy of drug loading [37]. Huang et al. presented a DNA-based origami biocompatible nanocarrier to aid delivery of the Ruthenium polypyridyl complexes (RuPOP) [38]. In the nude mice model, the effects of injecting RuPOP into tumor-bearing nude mice were observed. The results indicated that the DNA origami structures increased in vivo drug loading efficacy, as the nanosystems specifically targeted tumor sites while maintaining the healthy function of the organs.

An additional application of origami engineering in refining drug delivery systems is in the development of nanoscale robots. Li et al. presented a novel application of DNA origami for cancer therapy, developing a nanorobot that is designed to deliver thrombin only in the presence of the specified tumor [39]. The design includes an M13 bacteriophage genome DNA strand manipulated into a rectangular DNA sheet and secured by "staple" strands. This sheet is held in the shape of a hollow, cylindrical tube by DNA “fasteners.” Unlike other types of nanodevices, which are becoming increasingly complex and contain a large number of different parts, DNA origami structures consist of simple folds and hinges. The study investigated the efficacy of the nanorobotic system in delivering therapeutic thrombin to inhibit tumor growth in a melanoma mouse model, the nanorobot successfully targeted the primary tumor and prevented metastasis formation. Further research can focus on modifying nanostructure geometry for various treatments and applications.

\section{Microfluidics}

Origami has also become considered in designing 3-D microfluidic paper analytical devices ( $\mu$ PADs), which are used to determine the physical and chemical characteristics of fluids at a small scale using microchannels. Traditionally, 3-D $\mu$ PADs are fabricated by sequentially stacking 2-D layers of chromatography paper, though the principles of origami have been considered recently to develop a more efficient method of constructing 3-D geometries [40]. Liu and Crooks of the University of Texas at Austin discussed the assembly of 3-D microfluidic devices through paper folding, in which a single sheet of flat paper is transformed into a layered device [41]. The development for the resulting “oPads” was found to be advantageous over the typical sequential, layerby-layer construction of $\mu$ PADs, as fewer tools were needed, the oPads allowed for parallel colorimetric analysis of multiple substances, and adhesive tape was unnecessary, allowing for more efficient assembly and removing the need for laser cutting. A nine-layer device possessing photolithographically patterned reservoirs and channels was used to evaluate the efficacy of the origami assembly method and observe the flow of fluids. Aqueous solutions of rhodamine $6 \mathrm{G}$, erioglaucine, tartrazine, and erioglaucine mixed with tartrazine were injected into the device, and the resulting patterns indicated that the fluids separated into their respective chambers without mixing or nonspecific absorption.

Especially notable are the advancements of origami bioengineering in microsurgery. While current procedures might expose the patient to unnecessary risks due to the invasiveness of the surgery, foldable, self-actuated, and remote-controlled origami-inspired micro-robotic systems can perform tasks safely in the human body.

\section{Minimally invasive surgery}

Many patients experience the adverse effects of traditional surgeries, which entail the interference of surgeons and invasive medical devices when operating on a patient. Such procedures frequently result in perioperative complications, prolonged recoveries, and visible scarring [42]. These impediments, however, are greatly mitigated when doctors implement MIS, which requires few to no incisions. In comparing this approach with its more invasive counterpart, benefits included shorter hospital stays and lower readmission, complication, and mortality rates among Medicare patients receiving pancreatic and liver procedures of different levels of invasiveness [43].

Fortunately, surgical procedures have continued to become progressively less invasive during the past few decades in terms of dexterity enhancement and surgical assistance [1]. For example, capsule endoscopy has become an increasingly adopted method of gastrointestinal diagnosis, as it allows thorough yet unobtrusive inspection through the deployment of a miniature wireless camera encased in a dissolvable seal [44]. Another major advancement concerns the development of tetherless robotic surgical systems, which involve the navigation of miniature robots through the body's natural pathways. Biomedical microrobot systems can be characterized by their application [45], including targeted drug delivery [46], structural support [47], material removal [48], and telemetry [49]. 
As the dimensions of these surgical miniature robots correlate with the minimum size of the incision needed to operate them, progress in MIS has been aided by developments in microengineering and nanoengineering [50]. Though complications arise in attempting to further condense existing surgical technologies, origami-inspired tools have the potential to overcome traditional constraints; they can conveniently situate at a specified location, expand into functional three-dimensional structures, operate in confined spaces, and achieve a wide range of mobility [51], [5].

An especially critical application of origami engineering is in micro-robotics, specifically the incorporation of locomotive components into a foldable device, drawing inspiration from both nature and origami properties. Origami robots can morph into the desired structure through embedded actuators while still retaining a rigid structure through the folding pattern [52]. Referred to as 'robogami,' robotic systems incorporating origami properties can reconfigure from a two-dimensional sheet to an intricate three-dimensional structure and perform tasks in its environment, serving as an efficient method of robot fabrication [53], [54]. When incorporated with existing surgical tools and microbot systems, origami properties continue to advance the field. Their unique advantages stem from the microfabrication process, the self-actuation mechanisms, and the biocompatible materials used.

\section{Origami-Inspired Clinical Miniature Robots}

Miniature robots are small-scale devices that are highly relevant in microsurgery, having risen in prevalence tremendously over the past five years as compared to the past two decades. While their size allows for less invasive treatment, it also places limitations on possible actuation mechanisms [55], as studies developing miniature robots report dimensions ranging from $0.5-70 \mathrm{~mm}$. This section reviews the influence of origami engineering in biomedical miniature robots, highlighting specific devices from the past decade and comparing their material, dimensions, and applications (Table 2).

\section{Microgrippers}

Microgrippers are dexterous hand-shaped microrobots capable of imitating human-like hand motions including grasping objects, opening and closing, and other functions involving micromanipulation. Despite these abilities, their need for an external power source contributes to their unwieldy size, restricting their maneuverability in confined spaces and ability to navigate around human organs. Conventional microgrippers require connection to external wires or tubes and are driven by mechanical

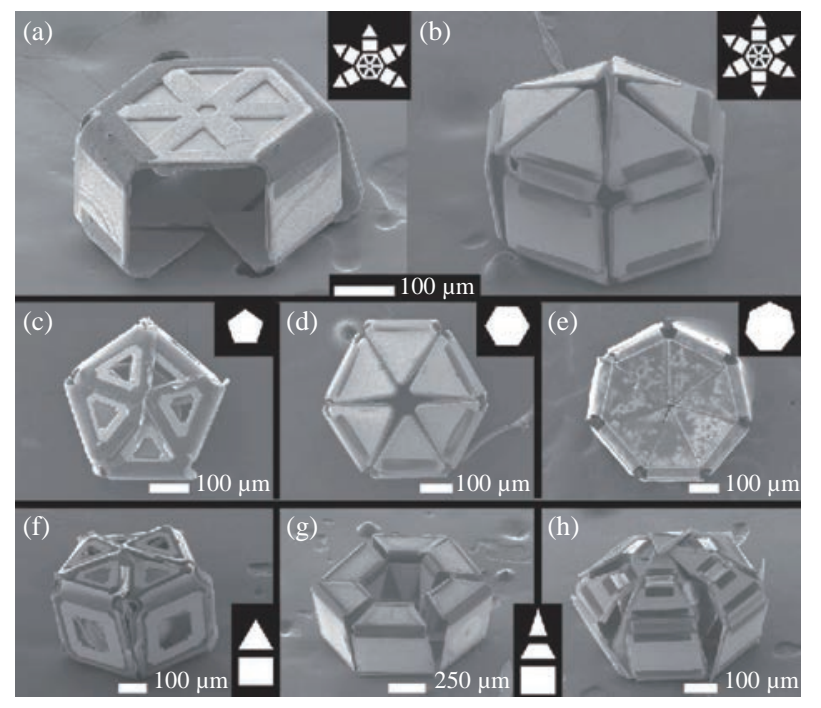

Fig. 4 Different designs of microgrippers, varying in the arrangement of digits around the central palm [57].

Table 2 Origami-inspired miniature robot designs with clinical applications in microsurgery

\begin{tabular}{|c|c|c|c|c|c|c|}
\hline Device & Material & Size & Applications & Actuation & References & Figure \\
\hline Microgripper & $\begin{array}{c}\text { Polymer and } \\
\text { bimetallic layers }\end{array}$ & $\begin{array}{c}700 \mu \mathrm{m} \text { (open) } \\
190 \mu \mathrm{m} \text { (closed) }\end{array}$ & $\begin{array}{c}\text { Biopsy } \\
\text { Cancer therapy }\end{array}$ & $\begin{array}{l}\text { Biochemical and } \\
\text { thermal actuation }\end{array}$ & $\begin{array}{l}\text { Leong, et al. } \\
\text { (2009) [57] }\end{array}$ & Fig. 4 \\
\hline Encapsulation robot & $\begin{array}{l}\text { Polystyrene and PVC } \\
\text { film }\end{array}$ & $1.7 \mathrm{~cm} \times 1.7 \mathrm{~cm}$ & Material delivery & $\begin{array}{l}\text { Global heating and } \\
\text { magnetic field }\end{array}$ & $\begin{array}{l}\text { Miyashita, et al. } \\
\text { (2015) [59] }\end{array}$ & Fig. 5 \\
\hline Gastrointestinal robot & $\begin{array}{l}\text { Dried pig intestine } \\
\text { and ice capsule }\end{array}$ & $\begin{array}{c}15 \mathrm{~mm} \times 30 \mathrm{~mm} \\
\times 5 \mathrm{~mm}\end{array}$ & Battery removal & Central magnet & $\begin{array}{l}\text { Miyashita, et al. } \\
\text { (2016) [60] }\end{array}$ & Fig. 6 \\
\hline Miniature Delta robot & Polyimide film & $\begin{array}{c}15 \mathrm{~mm} \times 15 \mathrm{~mm} \\
\times 20 \mathrm{~mm}\end{array}$ & Microsurgery & $\begin{array}{l}\text { Piezoelectric } \\
\text { actuators }\end{array}$ & $\begin{array}{l}\text { McClintock \& Wood } \\
\text { (2018) [63] }\end{array}$ & Fig. 7 \\
\hline $\begin{array}{l}\text { Teleoperated } \\
\text { manipulator }\end{array}$ & Piezoelectric ceramic & $\begin{array}{c}50 \mathrm{~mm} \times 70 \mathrm{~mm} \\
\times 50 \mathrm{~mm}\end{array}$ & Microsurgery & Three linear actuators & $\begin{array}{l}\text { Suzuki \& Wood } \\
\text { (2020) [65] }\end{array}$ & Fig. 8 \\
\hline
\end{tabular}




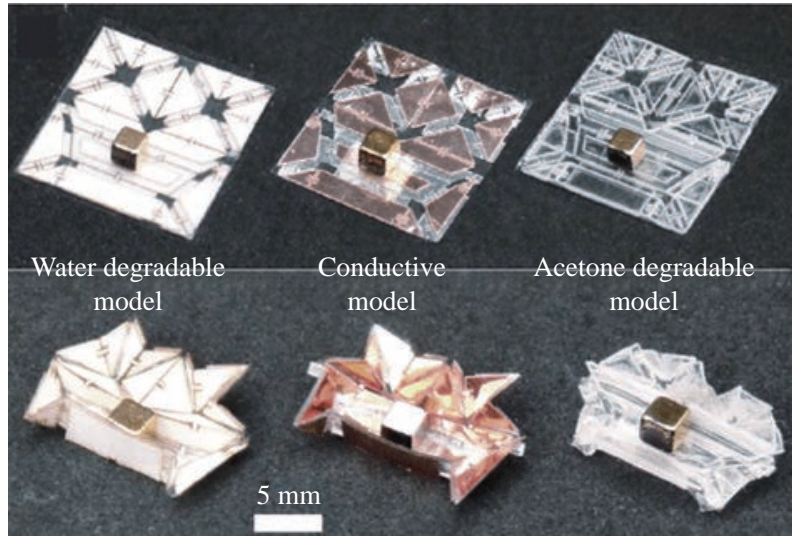

Fig. 5 Different models of encapsulation miniature robots, depending on design and material [59].

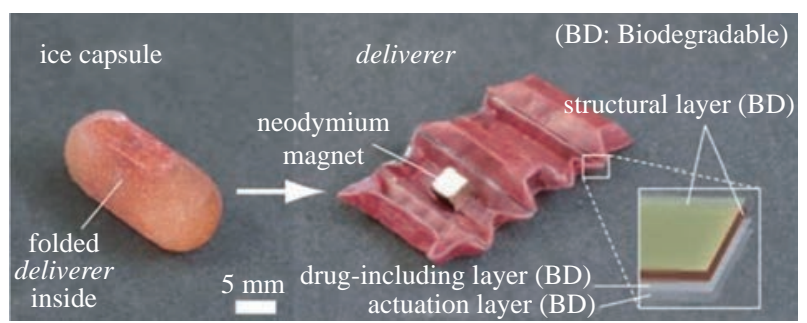

Fig. 6 Gastrointestinal origami-inspired robot in an ice capsule and an unfolded state [60].

or electrical signals, limiting their range of motion and potentially interfering with the normal physiological activities of the body. To address these limitations, there have been ongoing efforts in developing tetherless microgrippers, which are fabricated by the massproducible and scalable method of photolithography. Similarly to the crease patterns, folds, and faces that constitute projects in the field of origami engineering, tetherless microgrippers consist of rigid segments separated by flexible hinges. Their miniaturized scale allows them to possess a higher range of motion and can be remotely triggered, with promising applications in confined spaces such as the cardiovascular system, though there is still considerable development needed before implemented in clinical applications [56]. Leong et al. of John Hopkins University developed a tetherless microgripper that is actuated upon exposure to thermal or chemical conditions [57]. Through a combination of biochemical and thermal actuation, this gripper design can remove objects and perform biopsies in clinical settings. After examining several variants of microgripper designs constructed of polymer joints and ferromagnetic elements, grippers possessing six rotationally symmetric digits were found to be the most successful in retaining objects. Aside from evaluating the mechanical aspects of the microgripper, the study also explored the potential of thermally-triggered microgrippers in capturing clusters of live L929 fibroblast cells, and the results indicated that the retrieval process did not harm the cells. To evaluate the microgripper's performance in microsurgical applications, an in vitro biopsy on a bovine bladder tissue sample was conducted. While the claw easily traversed the smooth tissue, the claw phalanges became entangled and immobile when in contact with the rough tissue, requiring magnetic manipulation to be freed. Bassik et al. of John Hopkins University further expanded upon the concept of tetherless microgrippers, proposing modifications that would allow the device to autonomously reconfigure in a clinical environment, improving the efficacy of the treatment under biocompatible conditions [58]. By fabricating the device using a biopolymer material and pairing it with proteolytic enzymes secreted by cancer cells, the microgripper can be trained to react solely in (a)

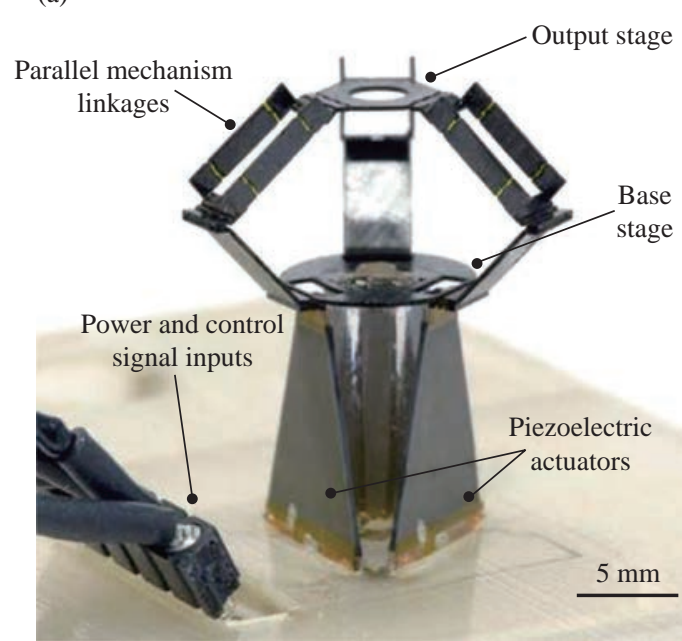

(b)

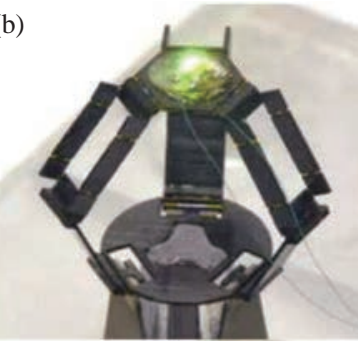

(d)

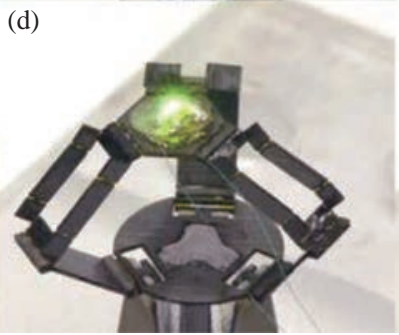

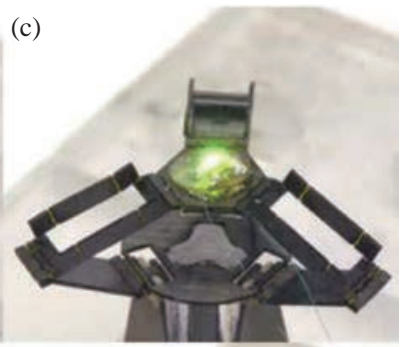

(e)

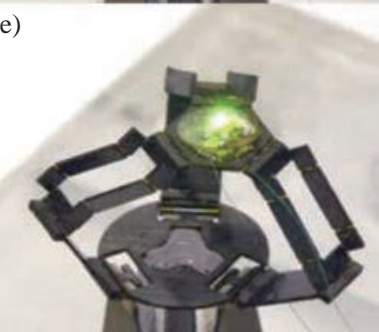

Fig. 7 Different perspectives of MilliDelta, with various orientations of linkages [63]. 

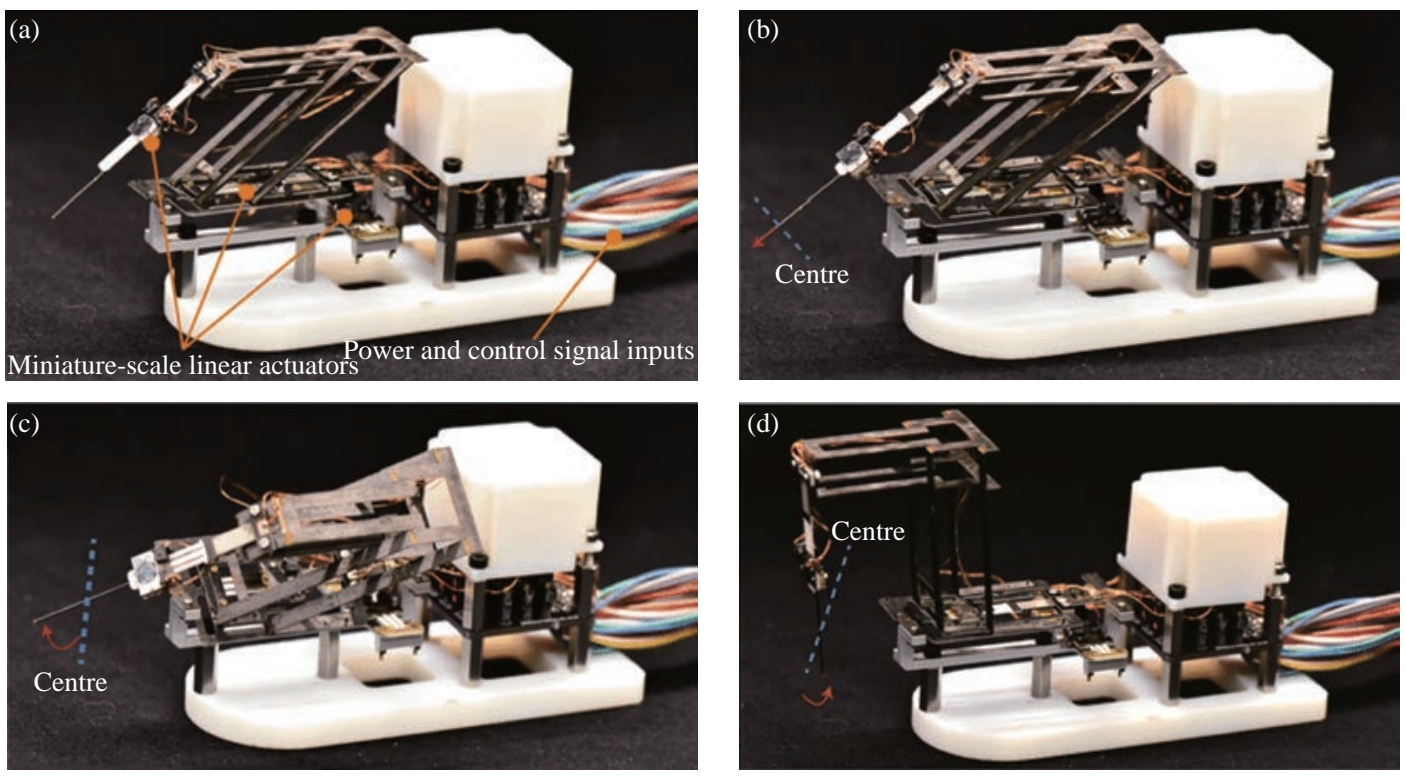

Fig. 8 Mini-RCM device demonstrating pop-up MEMS at different configurations [65].

target locations.

\section{Encapsulation robot}

Over the past decade, there has been an increasing demand for noninvasive, autonomous miniature robots capable of self-configuring on-site, as such devices are able to access and perform tasks in unreachable areas. In order to carry out this method of rapid fabrication, a notable approach involves transforming a fabricate planar sheet into the final 3-D structure. This class of printable robots can be fabricated more expediently and at lower costs compared to their traditional counterparts, and they also can be converted back to their planar state for the purposes of storage and transportation [13]. However, the need for simplicity in a self-folding robot introduces some challenges, including eliminating electronic parts, actuating the robot, and safely removing the robot from its environment. Miyashita et al. seeked to address these issues, proposing the development of a multi-crease, locomotive origami robot that can be fabricated with various materials and propelled without the inclusion of electronic components [59]. The microrobot consists of three distinct features: configuration, performance, and degradation. Composed of a threelayer sheet, consisting of a heat-sensitive contraction film sandwiched between two rigid structural layers, the robot maintains its structure while being bendable and flexible. Through global heating, the robot was demonstrated to successfully fold from a planar sheet into a three-dimensional figure that could climb, walk, jump, float, and pivot, controlled by an alternating magnetic field. The robot also completed a variety of tasks due to these mobile abilities, such as delivering a lightweight block, carrying two times its weight, and traversing rough terrain. Some difficulty was encountered in maintaining the robot's stability in response to an abrupt movement such as a sudden change in trajectory. Though in this study, the device was fabricated using polystyrene, PVC film, and silicone adhesive, which are dissolvable in acetone, replacing these materials with biodegradable counterparts would make the robot applicable in a clinical setting. While this study demonstrated the potential of printable robots in traversing terrain and performing basic tasks involving encapsulation and delivery, further research into alternate materials that are biocompatible is needed to evaluate the device's full potential in the biomedical field.

\section{Gastrointestinal robot}

Expanding on the previous study exploring different models of printable miniature robots for encapsulation [59], Miyashita et al. engineered an origami-inspired robot that is swallowed and activated in the stomach, where it can be remotely controlled to perform operations [60]. The main problem the paper addresses is the inadvertent swallowing of button batteries and resulting internal burns and risk of death, affecting thousands of individuals in the United States annually, mainly young children. Current interventional technologies for the gastrointestinal tract include capsule endoscopy, which involves swallowing an encapsulated wireless camera to visualize the internal 
lining [61], replacing traditional tethered endoscopes. While the proposed miniature robot does not possess electronic components and thus cannot visualize its surroundings, it can similarly be carried through the gastrointestinal tract. This study developed an ingestible robot that can safely remove a swallowed button battery, followed by another robot that addresses the damage, referred to as the "battery remover" and the "drug deliverer," respectively. The "battery remover” is encased in an ice layer, which melts soon after ingestion. Through magnetic attraction, the robot detaches the battery from the stomach's wall and then safely passes through the gastrointestinal tract. Subsequently, the "drug deliverer" is swallowed and is guided to the inflammation site, where it administers a drug. Unlike the more simple features of the battery remover, the drug deliverer consists of origami accordion-style folds, which, upon deployment, expand significantly. Encased in biodegradable pig intestinal walls, the drug deliverer travels by "rolling” and is propelled through controlled magnetic pulses. To demonstrate proof-of-concept results of the robot's ability to be deployed to the site, remove the battery, and patch the wound, a simulation involving an silicone gastrointestinal tract with an artificial mucous membrane was conducted. First, the ice capsule containing the "battery remover" robot was guided through the "esophagus” into the "stomach,” and the dissolution time varied depending on the water temperature. Then, the ice capsule connected to the battery, and both were discharged out the tract. Finally, the ice capsule containing the "drug deliverer" robot was transported to the stomach, where it tumbled and walked to the site of the artificial ulcer. This study offered valuable insight into the basic mechanics of an ingestible miniature self-folding robot and a glimpse into its potential in a real-world clinical environment. While the artificial stomach lining served as an appropriate representation of a patient's internal organ, other conditions including temperature, movement, acidity, and other uncertainties were not taken into account. Research that further attempts to closely imitate bodily conditions would more accurately simulate the route of the miniature robot through an individual's gastrointestinal tract and evaluate its ability to safely remove a button battery and dissolve into its environment.

\section{Miniature delta robot}

Over the past several years, there has been significant development in the field of surgical robotics, specifically in robotic surgery devices that are able to assist surgeons and improve upon existing practices in traditional minimally invasive surgery [62]. One such robot is the Delta robot, a mechanism consisting of parallel manipulators capable of tasks including microsurgery. Delta robots are composed of two parallel plates-an output stage and a base-attached together by three kinematic linkages, and these lightweight arms are able to control the moving output stage in a fast and precise manner. While the scale of Delta robots had been gradually reduced over time, further shrinking the device down to the millimeter scale had proved challenging. McClintock et al. at the Wyss Institute at Harvard University were able to overcome this issue of miniaturization, designing an origami-inspired millimeter-scale Delta robot known as the "milliDelta” using pop-up MEMS manufacturing [63]. The Delta robot is a 3-DOF (degrees of freedom) parallel mechanism design that is widely used in industrial processes; on a small scale, the Delta robot is especially effective in micro-manipulation and micro-assembly. The piezoelectric actuators allow the milliRobot to perform high-frequency movements, and in a clinical setting, the robot can be used for tremor compensation, reducing hand tremors by $81 \%$. Moreover, it was shown that the milliDelta can operate in an area of seven cubic millimeters, still achieving high precision at frequencies up to 25 times higher than conventional Delta robots. With regards to potential clinical applications, the milliDelta can be developed to supplement existing robotic devices for operations such as retinal microsurgeries, or it can also serve as standalone apparatus for examining cells in research laboratories. While the milliDelta was established as an effective mechanism for micromanipulation tasks, future research can be conducted with the goal of further reducing the scale of the device.

\section{Teleoperated manipulator}

As demonstrated in the previous study exploring the implementation of a miniaturized Delta robot [63], research in robot-assisted surgery is making breakthroughs in the field of minimally invasive surgery. An example of this is in laparoscopic surgery, in which the surgeon inserts tools and a small camera into the patient's body through incisions [64]. While current robot systems have proven helpful in operating rooms due to their ability to perform with precision and flexibility, their large size affects their agility and handling of delicate internal human structures. Suzuki and Wood from the Wyss Institute at Harvard 
University developed a noninvasive, origami-inspired miniature manipulator known as the "mini-RCM" (remote center of motion) using an origami-inspired Pop-Up MEMS manufacturing technique [65]. The mini-RCM utilizes three linear actuators (mini-LAs) to accurately control a surgical tool, and deviations are corrected for by the LED-based optical sensors that are integrated into the mini-LAs. Moreover, the mini-RCM's small size allows it to be easily installed and removed. To evaluate the precision of the miniRCM, a human operator traced a tiny square using a ballpoint pen with and without assistance from the device; when using the mini-RCM, error was reduced by $68 \%$. The efficacy of the mini-RCM was also tested in a simulated clinical environment in which a surgeon performed mock retinal vein cannulation. The needle successfully punctured the "retinal vein," represented by a fabricated silicone tube of the same size, without causing local damage. To further validate implementation of the mini-RCM in clinical applications, procedures simulating retinal microsurgery should also consider external forces by accounting for the viscosity of the vitreous and applying a reasonable amount of force to the sclera, for instance.

\section{Challenges and Opportunities}

In the past ten years, the application of origami properties has made progress in the areas of microsurgery with regards to improving precision, minimizing scale, and overall reducing postoperative complications and improving a patient's wellbeing. This section describes the complications associated with prototype design, material selection, fabrication, and deployment and self-actuation. In addition, this section describes potential approaches for these challenges and presents future opportunities for advancement.

\section{Design}

Much of origami engineering relies on developing the appropriate crease pattern according to how effectively it will ultimately transfigure into its final 3-D structure. Usually, folding patterns are unidirectional in that creases are not reprogrammable after folded and must remain in their assigned state, either as a mountain or a valley fold. Attempting to change one to the other may place mechanical strain on the hinge, weakening the structural integrity of the device [66]. Therefore, extensive planning must be conducted during the design stage before the planar sheet is fabricated, typically using graphical software intended for origami design. One such example is TreeMaker [67], a computer program developed by Robert Lang which implements the tree method. By inputting a tree graph, the designer is able to create a uniaxial base, which refers to a base in which all flaps can be collapsed and lie along a single axis, to which all of its hinge creases are perpendicular [68]. Another useful software program is known as Origamizer. a design program that implements an algorithm intended for the folding of paper into complex polyhedral models. Origamizer examines a model's surface mesh, a representation consisting of edges, vertices, and faces, and divides it into triangular sections, resulting in a planar triangular mesh [69]. While Treemaker produces shapes based on a uniaxial base, Origamizer creates meshed surfaces; both programs result in shapes with unique geometric characteristics.

\section{Material}

Depending on the application and design, different amounts of strain energy stored in the creases will be required. Creases can be referred to by their hinge index, which can be increased through deeper perforations, and materials with a low hinge index can store higher amounts of strain energy in the creases [26]. As the fundamental properties of origami assume close to a near-zero-thickness material, the thickness of the more hefty engineering materials used must also be taken into consideration [70].

Unlike other applications of origami properties such as space exploration and structural engineering, the biomedical field has the unique aspect of being closely interrelated with living organisms. As a result, researchers must be even more selective in their materials and design, as the resulting products have the potential to directly affect lives (Table 3). The development of such types of substances marked a significant breakthrough in the medical field, as previously, treatments had been limited to highly invasive and risky surgery [71].

\section{Fabrication}

Fabricating a foldable robot on the micro- or nanoscale can be challenging without the proper techniques, which include high-resolution lithography patterning, plasma-based pattern transfer, and thin film deposition processes [77]. Furthermore, implementing miniature robots in a clinical environment entails the device follow certain requirements to be able to operate safely 


\begin{tabular}{|c|c|c|c|c|}
\hline Category & Biomaterial & Description & Biomedical Applications & References \\
\hline \multirow{5}{*}{ Metallic } & Stainless steels & $\begin{array}{l}\text { Low cost, corrosion-resistant } \\
\text { Nickel-free high nitrogen austenitic stainless steels; } \\
\text { nickel-free alternative, avoids adverse effect of Ni ions }\end{array}$ & $\begin{array}{l}\text { Surgical instruments } \\
\text { Finger prostheses }\end{array}$ & {$[72]$} \\
\hline & Titanium-based alloys & Strong, low modulus of elasticity, high biocompatibility & $\begin{array}{l}\text { Bone fixation plates, } \\
\text { hip joints }\end{array}$ & [73] \\
\hline & Magnesium alloys & $\begin{array}{c}\text { Biodegradable; dissolvable } \mathrm{Mg}^{2+} \text { consumed by body assists in } \\
\text { healing process }\end{array}$ & $\begin{array}{l}\text { Cardiovascular stents, } \\
\text { orthopedic implants }\end{array}$ & {$[74]$} \\
\hline & Cobalt-based alloys & High corrosion, wear resistance, low friction coefficient & Joint prosthesis & {$[75]$} \\
\hline & Shape-memory alloys & $\begin{array}{l}\text { Able to retain memory of its original shape and return } \\
\text { to it after being deformed }\end{array}$ & Stents, braces, staples & [14] \\
\hline \multirow[t]{4}{*}{$\begin{array}{c}\text { Inorganic and } \\
\text { non-metallic }\end{array}$} & Alumina \& Zirconia & $\begin{array}{l}\text { High temperature and wear resistance, } \\
\text { wettability, low friction }\end{array}$ & $\begin{array}{l}\text { Joint prosthesis, ceramic } \\
\text { implants }\end{array}$ & [75] \\
\hline & Soft \& hard tissues & $\begin{array}{l}\text { "Soft” refers to muscles, skin, and fat, and other organ tissues } \\
\text { "Hard" includes bones, enamel, cementum, and dentin }\end{array}$ & Orthopedic surgery & {$[76]$} \\
\hline & & & & \\
\hline & Polymers & $\begin{array}{l}\text { Viscoelastic behavior, low thermal conductivity } \\
\text { Below glass transition temperature }\left(\mathrm{T}_{\mathrm{g}}\right) \text {, polymer changes } \\
\text { from soft and rubbery to a stiff glassy state }\end{array}$ & Knee joint replacement & [75] \\
\hline
\end{tabular}

and efficiently.

Miniaturizing origami robots to the scale required in biomedical applications requires overcoming numerous challenges, mainly in fabricating the device and ensuring self-actuation at a small scale [78], [45]. A process that can greatly ease this transformation is microfabrication, which, in the context of microbiology, refers to the usage of microtechnology at even a cellular level [79]. This process is the foundation of origami-inspired microrobots, as it ensures that the robot performs efficiently at a small scale. Forming creases in the material can be achieved through computer numeric controlled (CNC) manufacturing methods, such as laser cutting, incremental sheet folding, and abrasive water jet cutting [26].

Recently, the integration of origami principles with traditional microfabrication methods for medical devices and microrobots has been discussed. Zhu et al. of the University of Michigan proposed an electrothermal micro-origami system that can actuate without the assistance of an external stimulus. Layers of polymer and gold serve as onboard actuators, though current models still require a tether. With the aid of a microcontroller to supply electric current to the system, the microrobot will be able to move autonomously with less restrictive movements than their traditional counterparts [80].

\section{Deployment and self-actuation}

There are several viable assembly methods for origami-based designs, though the two leading approaches are using automated machinery to create the final structure or developing self-folding designs [26]. Self-folding can be achieved through the use of smart material actuators that are capable of autonomously folding into a functional threedimensional structure and are distributed throughout an origami sheet [52].

While large-scale origami typically requires motors and springs to operate, small-scale origami utilizes a mechanism similar to shape-programmable matter, which can reconfigure themselves into the desired structure based on input [81]. The sheet consists of a shape-memory material, which is unique in its ability to adopt a particular structure; while most materials generally after being deformed gradually return to their original state, shape-memory materials revert only when exposed to the appropriate stimulus [82]. In origami-based robotic design, this stimulus is often conveyed via temperature variation. When exposed to 
Table 4 Comparison of shape-memory alloys and polymers

\begin{tabular}{|c|c|c|}
\hline & Shape-memory alloys (SMAs) & Shape-memory polymers (SMPs) \\
\hline Description & $\begin{array}{l}\text { Alloys that are trained at high temperatures to } \\
\text { retain a specified structure. }\end{array}$ & $\begin{array}{l}\text { Polymers that reconfigure in response to an external } \\
\text { stimulus, typically via global heating. }\end{array}$ \\
\hline Examples & $\begin{array}{l}\text { Nickel-titanium (NiTi) is predominant in the biomedical field, as it } \\
\text { possesses both thermal shape memory and superelasticity [54]. }\end{array}$ & $\begin{array}{l}\text { Polyolefin, polyvinyl chloride, and polystyrene } \\
\text { demonstrate unidirectionality for one-time assembly. }\end{array}$ \\
\hline Advantages & $\begin{array}{l}\text { Can be used in both its planar and coiled wire } \\
\text { forms to actuate assembly and movement. }\end{array}$ & $\begin{array}{l}\text { Inexpensive and possess a multiple-shape memory effect; the } \\
\text { material is capable of "memorizing" numerous designs [83]. }\end{array}$ \\
\hline Disadvantages & $\begin{array}{l}\text { A high training temperature is required, } \\
\text { and using metal as a material might be challenging. }\end{array}$ & $\begin{array}{l}\text { Generally not bidirectional, meaning that an external force is } \\
\text { required to reset the shape of the polymer after actuation. }\end{array}$ \\
\hline
\end{tabular}

uniform heating, the material contracts to the desired three-dimensional structure at predetermined angles, transitioning between the martensite and austenite phases [26]. Shape-memory alloys and shapememory polymers are at the forefront among materials stimulated by global heating and used in self-assembly for origami-based designs [52] (Table 4).

Another approach for self-assembly used in minimally invasive robots consists of piezoelectric materials. Unlike shape-memory alloys and polymers, which respond to changes in temperature, piezoelectric materials change response when exposed to an applied electrical charge and are especially efficient at the millimeter scale [52].

\section{Conclusions}

Despite being an ancient art not commonly associated with engineering projects, origami folding is providing valuable insight into the biomedical field. More specifically, extrapolating the origami properties of rigid-foldability and pliability has helped produce more efficient designs for clinical use. Implementation of origami engineering in noninvasive microsurgery has been progressing throughout the past decade, as evident in recent notable developments in origamiinspired microsurgical robotic systems, which improve upon their conventional counterparts by further simplifying and miniaturizing the designs. While the small-scale conditions might present a challenge and reduce the capabilities of the miniature robot, advancements in fabrication, advancements in microfabrication methods will continue to pave the way for future applications in microsurgery.

\section{Conflict of Interests}

The authors declare that no competing interest exists.

\section{References}

[1] M.J. Mack, Minimally Invasive and Robotic Surgery. JAMA Network Open, 2001, 285(5): 568-572.

[2] K.H. Sheetz, J. Claflin, J.B. Dimick, Trends in the Adoption of Robotic Surgery for Common Surgical Procedures. JAMA, 2020, 3(1): 259-267.

[3] C.D. Onal, D. Rus, A modular approach to soft robots. 2012 4th IEEE RAS \& EMBS International Conference on Biomedical Robotics and Biomechatronics. Rome, Italy, Jun. 24-27, 2012: 1038-1045.

[4] M. Schenk, S.D. Guest, Origami Folding: A Structural Engineering Approach. Fifth International Meeting of Origami Science, Mathematics, and Education. Singapore, Jul. 13-17, 2010: 291-303.

[5] N. Turner, B. Goodwine, M. Sen, A review of origami applications in mechanical engineering. Proceedings of the Institution of Mechanical Engineers, Part C: Journal of Mechanical Engineering Science, 2016, 230(14): 23452362.

[6] R.J. Lang, The Complete Book of Origami: Step-by-Step Instructions in Over 1000 Diagrams. Dover Publications, 2012: 1-2.

[7] S. Chen, J. Chen, X. Zhang, et al., Kirigami/ origami: unfolding the new regime of advanced 3D microfabrication/nanofabrication with "folding". Light: Science \& Applications, 2020, 9:75.

[8] M. Frishberg, Origami-Inspired Engineering: Fold, Bend, Design, Build. Research Technology Management, 2016, 59(3): 6-7.

[9] K. Liapi, W. Wang, G. Jenkins, Transformable Architecture Inspired by the Origami Art: Computer Visualization as a Tool for Form Exploration. Proceedings of the 2002 Annual Conference of the Association for Computer Aided Design in Architecture. Pomona, CA, USA, Oct. 24-27, 2002: 381-388.

[10] S. Li, H. Fang, S. Sadeghi, et al., Architected Origami Materials: How Folding Creates Sophisticated Mechanical Properties. Advanced Materials, 2018, 31(5): e1805282.

[11] Z. Song, T. Ma, R. Tang, et al., Origami lithium-ion batteries. Nature Communications, 2014, 5: 3140.

[12] S. Zirbel, R. Lang, M. Thomson, et al., Accommodating Thickness in Origami-Based Deployable Arrays. Journal of Mechanical Design, 2013, 135(11): 111005.

[13] C.D. Onal, R.J. Wood, D. Rus, Towards printable robotics: Origami-inspired planar fabrication of three-dimensional mechanisms. 2011 IEEE International Conference on Robotics and Automation. Shanghai, China, May 9-13, 2011: 4608-4613.

[14] K. Kuribayashi, K. Tsuchiya, Z. You, et al., Selfdeployable origami stent grafts as a biomedical application of Ni-rich TiNi shape memory alloy foil. Materials Science and Engineering: A, 2005, 419(1-2): 131-137.

[15] J.F.V. Vincent, Deployable Structures in Nature: Potential 
for Biomimicking. Proceedings of the Institution of Mechanical Engineers, Part C: Journal of Mechanical Engineering Science, 2000, 214(1): 1-10.

[16] S. Yao, X. Liu, S. Georgakopoulos, et al., A novel reconfigurable origami spring antenna. 2014 IEEE Antennas and Propagation Society International Symposium. Memphis, TN, USA, Jul. 6-11, 2014: 374375.

[17] Y. Chen, J. Yan, J. Feng, et al., Geometric and Kinematic Analyses and Novel Characteristics of Origami-Inspired Structures. Symmetry, 2019, 11(9): 1101.

[18] S.J.P. Callens, A.A. Zadpoor, From flat sheets to curved geometries: Origami and kirigami approaches. Materials Today, 2018, 21(3): 241-264.

[19] S.W. Grey, F. Scarpa, M. Schenk, Mechanics of paperfolded origami: A cautionary tale. Mechanics Research Communications, 2020, 107: e103540.

[20] W. Wu, Z. You, A solution for folding rigid tall shopping bags. Proceedings of the Royal Society A: Mathematical, Physical and Engineering Sciences, 2011, 467: 25612574.

[21] R. Konings, R. Thijs, Foldable Containers: A New Perspective on Reducing Container-Repositioning Costs. European Journal of Transport and Infrastructure Research, 2001, 1(4).

[22] N. Cheng, A. Rodriguez, A. Koger, Folded Sun-Shades: from Origami to Architecture. Proceedings of the 100th Annual ACSA National Meeting. Boston, MA, USA, Mar. 1-4, 2012: 250-257.

[23] A.P. Thrall, C.P. Quaglia, Accordion shelters: A historical review of origami-like deployable shelters. Engineering Structures, 2014, 59: 686-692.

[24] Y. Nishiyama, Miura Folding: Applying Origami to Space Exploration. International Journal of Pure and Applied Mathematics, 2012, 79: 269-279.

[25] J. Paik, B.H. Shin, Y. Bang, et al., Development of an Anthropomorphic Robotic Arm and Hand for Interactive Humanoids. Journal of Bionic Engineering, 2012, 9(2): 133-142.

[26] M. Johnson, Y. Chen, S. Hovet, et al., Fabricating biomedical origami: a state-of-the-art review. International journal of computer assisted radiology and surgery, 2017, 12(11): 2023-2032.

[27] M. Gottsauner-Wolf, D.J. Moliterno, A.M. Lincoff, et al., Restenosis-an open file. Clinical Cardiology, 1996, 19: 347-356.

[28] A.J. Taylor, Y. Chen, M.P. Fok, et al., Cardiovascular Catheter with an Expandable Origami Structure. Journal of Medical Devices, 2017, 11(3): 034505.

[29] K. Chinzei, R. Kikinis, F.A. Jolesz, MR Compatibility of Mechatronic Devices: Design Criteria. Medical Image Computing and Computer Assisted Intervention. Cambridge, UK, Sept. 19-22, 1999: 1020-1030.

[30] S.T. Wang, M.A. Gray, S. Xuan, et al., DNA origami protection and molecular interfacing through engineered sequence-defined peptoids. Proceedings of the National Academy of Sciences of the United States of America, 2020, 117(12): 6339-6348.

[31] E. Tasciotti, Smart cancer therapy with DNA origami. Nature Biotechnology, 2018, 36(3): 234-235.

[32] R. Fernandes, D. Gracias, Self-folding polymeric containers for encapsulation and delivery of drugs. Advanced Drug Delivery Reviews, 2012, 64(14): 15791589.

[33] T.S. Shim, S.H. Kim, C.J. Heo, et al., Controlled Origami Folding of Hydrogel Bilayers with Sustained Reversibility for Robust Microcarriers. Angewandte Chemie International Edition, 2012, 51: 1420-1423.

[34] H. He, J. Guan, J.L. Lee, An oral delivery device based on self-folding hydrogels. Journal of Controlled Release,
2006, 110(2): 339-346.

[35] Y.V. Kalinin, J.S. Randhawa, D.H. Gracias, ThreeDimensional Chemical Patterns for Cellular SelfOrganization. Angewandte Chemie International Edition, 2011, 50: 2549-2553.

[36] U. Ndagi, N. Mhlongo, M.E. Soliman, Metal complexes in cancer therapy-an update from drug design perspective. Drug Design, Development, and Therapy, 2017, 11: 599616.

[37] A.S. Gonçalves, A.S. Macedo, E.B. Souto, Therapeutic nanosystems for oncology nanomedicine. Clinical and Translational Oncology, 2012, 14(12): 883-890.

[38] Y. Huang, W. Huang, L. Chan, et al., A multifunctional DNA origami as carrier of metal complexes to achieve enhanced tumoral delivery and nullified systemic toxicity. Biomaterials, 2016, 103: 183-196.

[39] S. Li, Q. Jiang, S. Liu, et al., A DNA nanorobot functions as a cancer therapeutic in response to a molecular trigger in vivo. Nature Biotechnology, 2018, 36: 258-264.

[40] A.W. Martinez, S.T. Phillips, G.M. Whitesides, Threedimensional microfluidic devices fabricated in layered paper and tape. Proceedings of the National Academy of Sciences of the United States of America, 2008, 105(50): 19606-19611.

[41] H. Liu, R.M. Crooks. Three-dimensional paper microfluidic devices assembled using the principles of origami. Journal of the American Chemistry Society, 2011, 133(44): 17564-17566.

[42] D.B. Jones, R.V. Rege, Minimally Invasive Surgery. Surgical Research. Elsevier Inc., 2001: 573-582.

[43] Q. Chen, K. Merath, F. Bagante, et al., A Comparison of Open and Minimally Invasive Surgery for Hepatic and Pancreatic Resections Among the Medicare Population. Journal of gastrointestinal surgery: official journal of the Society for Surgery of the Alimentary Tract, 2018, 22(12): 2088-2096.

[44] J.F. Rey, H. Ogata, N. Hosoe, et al., Feasibility of stomach exploration with a guided capsule endoscope. Endoscopy, 2010, 42(7): 541-545.

[45] B.J. Nelson, I.K. Kaliakatsos, J.J. Abbott, Microrobots for Minimally Invasive Medicine. Annual Review of Biomedical Engineering, 2010, 12(1): 55-85.

[46] G. Dogangil, O. Ergeneman, J.J. Abbott, et al., Toward targeted retinal drug delivery with wireless magnetic microrobots. IEEE/RSJ International Conference on Intelligent Robots and Systems. Nice, France, Sept. 2226, 2008: 1921-1926.

[47] A.C. Jones, B. Milthorpe, H. Averdunk, et al., Analysis of 3D bone ingrowth into polymer scaffolds via microcomputed tomography imaging. Biomaterials, 2004, 25(20): 4947-4954.

[48] S. Byun, J.M. Lim, S.J. Paik, et al., Barbed micro-spikes for micro-scale biopsy. Journal of Micromechanics and Microengineering, 2005, 15(6): 1279-1284.

[49] M. Fluckiger, B.J. Nelson, Ultrasound emitter localization in heterogeneous media. $29^{\text {th }}$ Annual International Conference of the IEEE Engineering in Medicine and Biology Society. Lyon, France, Aug. 22-26, 2007: 28672870.

[50] C.L. Randall, E. Gultepe, D.H. Gracias, Self-folding devices and materials for biomedical applications. Trends in Biotechnology, 2011, 30(3): 138-146.

[51] R. Fernandes, D.H. Gracias, Toward a miniaturized mechanical surgeon. Materials Today, 2009, 12(10): 1420.

[52] D. Rus, M. Tolley, Design, fabrication and control of origami robots. Nature Reviews Materials, 2018, 3(6): 101-112.

[53] A. Firouzeh, Y. Sun, H. Lee, et al., Sensor and actuator integrated low-profile robotic origami. IEEE/RSJ 
International Conference on Intelligent Robots and Systems. Tokyo, Japan, Nov. 3-7, 2013: 4937-4944.

[54] A. Firouzeh, J. Paik, Robogami: A Fully Integrated LowProfile Robotic Origami. Journal of Mechanisms and Robotics, 2015, 7(2): 021009.

[55] M. Koleoso, X. Feng, Y. Xue, et al., Micro/nanoscale magnetic robots for biomedical applications. Materials Today Bio, 2020, 8: 100085.

[56] C. Yin, F. Wei, Z. Zhan, et al., Untethered microgripperthe dexterous hand at microscale. Biomedical Microdevices, 2019, 21(4): 1-18.

[57] T.G. Leong, C.L. Randall, B.R. Benson, et al., Tetherless Thermobiochemically Actuated Microgrippers. National Academy of Sciences, 2009, 106(3): 703-708.

[58] N. Bassik, A. Brafman, A.M. Zarafshar, et al., Enzymatically Triggered Actuation of Miniaturized Tools. Journal of the American Chemical Society, 2010, 132(46): 16314-16317.

[59] S. Miyashita, S. Guitron, M. Ludersdorfer, et al., An untethered miniature origami robot that self-folds, walks, swims, and degrades. IEEE International Conference on Robotics and Automation. Seattle, WA, USA, May 26-30, 2015: 1490-1496.

[60] S. Miyashita, S. Guitron, K. Yoshida, et al., Ingestible, controllable, and degradable origami robot for patching stomach wounds. Proceedings of the IEEE International Conference on Robotics and Automation. Stockholm, Sweden, May 16-21, 2016: 909-916.

[61] G.S. Lien, C.W. Liu, J.A. Jiang, et al., Magnetic control system targeted for capsule endoscopic operations in the stomach - design, fabrication, and in vitro and ex vivo evaluations. IEEE Transactions on Biomedical Engineering, 2012, 59(7): 2068-2079.

[62] D. Pisla, B. Gherman, C. Vaida, et al., An active hybrid parallel robot for minimally invasive surgery. Robotics and Computer-Integrated Manufacturing, 2013, 29(4): 203-221.

[63] H. McClintock, F.Z. Temel, N. Doshi, et al., The milliDelta: A high-bandwidth, high-precision, millimeterscale Delta robot. Science Robotics, 2018, 3(14).

[64] K.J. Huang, K.T. Lin, C.J. Wu, et al., Single incision laparoscopic surgery using conventional laparoscopic instruments versus two-port laparoscopic surgery for adnexal lesions. Scientific Reports, 2021, 11: 4118.

[65] H. Suzuki, R.J. Wood, Origami-inspired miniature manipulator for teleoperated microsurgery. Nature Machine Intelligence, 2020, 2: 437-446.

[66] B. Treml, A. Gillman, P. Buskhol, et al., Origami mechanologic. Proceedings of the National Academy of Sciences of the United States of America, 2018, 115(27): 6916-6921.

[67] R.J. Lang, A computational algorithm for origami design. Proceedings of the twelfth annual symposium on computational geometry. Philadelphia, PA, USA, May 2426, 1999: 98-105.

[68] A. Bateman, Computer Tools and Algorithms for Origami Tessellation Design. Origami 3: Third International Meeting of Origami Mathematics, Science, and Education. Asilomar, CA, USA, Mar. 9-11, 2002: 121.

[69] E. Demaine, T. Tachi, Origamizer: A Practical Algorithm for Folding Any Polyhedron. The 33rd International Symposium on Computational Geometry. Brisbane, Australia, Jul. 4-7, 2017: 34:1-34:16.
[70] S.A. Zirbel, R.J. Lang, M.W. Thomson, et al., Accommodating Thickness in Origami-Based Deployable Arrays. Journal of Mechanical Design, 2013, 135(11): 111005.

[71] D. Axinte, Y.B. Guo, N. Sugita, et al., Machining of biocompatible materials-Recent advances. CIRP Annals, 2019, 68(2): 629-652.

[72] M. Talha, C.K. Behera, O.P. Sinha, A review on nickelfree nitrogen containing austenitic stainless steels for biomedical applications. Materials Science and Engineering: C, 2013, 33(7): 3563-3575.

[73] L.T. Kuhn, (Biomaterials) Introduction to Biomedical Engineering (Third Edition). Elsevier Inc., 2012: 219271.

[74] P.K. Chu, G.S. Wu, (Surface design of biodegradable magnesium alloys for biomedical applications) Surface Modification of Magnesium and its Alloys for Biomedical Applications (Volume 1: Biological Interactions, Mechanical Properties and Testing). Woodhead Publishing, 2015: 89-119.

[75] R. Tsukamoto, S. Chen, T. Asano, et al., Improved wear performance with crosslinked UHMWPE and zirconia implants in knee simulation. Acta Orthopaedica, 2006, 77(3): 505-511.

[76] M. Mitsuishi, J. Cao, P. Bártolo, et al., Biomanufacturing. CIRP Annals, 2013, 62(2): 585-606.

[77] T. Betancourt, L. Brannon-Peppas, Micro- and nanofabrication methods in nanotechnological medical and pharmaceutical devices, International Journal of Nanomedicine, 2006, 1(4): 483-495.

[78] M.Z. Miskin, K.J. Dorsey, B. Bircan, et al., Graphenebased bimorphs for micro-sized, autonomous origami machines. Proceedings of the National Academy of Sciences of the United States of America, 2018, 115(3): 466-470.

[79] D.B. Weibel, W.R. DiLuzio, G.M. Whitesides, Microfabrication meets microbiology. Nature Reviews Microbiology, 2007, 5(3): 209-218.

[80] Y. Zhu, M. Birla, K.R. Oldham, et al., Elastically and plastically foldable electrothermal micro-origami for controllable and rapid shape morphing. Advanced Functional Materials, 2020, 30(40): e2003741.

[81] E. Hawkes, B. An, N. Benbernou, et al., Programmable matter by folding. Proceedings of the National Academy of Sciences of the United States of America, 2010, 107(28): 12441-12445.

[82] W.M. Huang, C.L. Song, Y.Q. Fu, et al., Shaping tissue with shape memory materials. Advanced Drug Delivery Reviews, 2013, 65(4): 515-535.

[83] T. Xie, Tunable polymer multi-shape memory effect. Nature, 2010, 464: 267-270.

[84] M.R. Garza, E.A. Peraza-Hernandez, D.J. Hartl, Self-folding origami surfaces of non-zero Gaussian curvature. Proc. SPIE 10968, Behavior and Mechanics of Multifunctional Materials XIII. Denver, CO, USA, March 3-7, 2019.

Copyright $@$ Vienna Parnell. This is an open-access article distributed under the terms of the Creative Commons Attribution License, which permits unrestricted use, distribution, and reproduction in any medium, provided the original author and source are credited. 